\title{
Generation of electromagnetic pulses from plasma channels induced by femtosecond light strings
}

\author{
Chung-Chieh Cheng, E. M. Wright, and J. V. Moloney \\ Arizona Center for Mathematical Sciences, and Optical Sciences Center, Tucson, AZ 85721, USA
}

(October 27, 2018)

We present a model that elucidates the physics underlying the generation of an electromagnetic pulse from a femtosecond laser induced plasma channel. The radiation pressure force from the laser pulse spatially separates the ionized electrons from the heavier ions and the induced dipole moment subsequently oscillates at the plasma frequency and radiates an electromagnetic pulse.

33.80.Wz,42.65.Re,52.40.Db

Recent investigations of the propagation of intense femtosecond infrared (IR) laser pulses in air show that the dynamical interaction between nonlinear self-focusing, plasma defocusing, and group-velocity dispersion can cause an initial beam to break up spatially into several filaments, or light strings, with diameters around a hundred microns that can maintain themselves over long distances [1] 5]. It has been observed experimentally that femtosecond light strings in turn produce plasma channels by multi-photon ionization (MPI) along their direction of propagation with lengths on the order of centimeters 6 10]. Observations of the electromagnetic pulses (EMPs) from light string induced plasmas suggest that these channels attain dipole moments during the laser pulse which subsequently oscillate at the plasma frequency and radiate [10].

In this letter we present a simple model for EMP generation from femtosecond light string induced plasmas to elucidate the underlying physics, in particular how the initial dipole moment is produced in the plasma channel, and the characteristic field strengths and spectrum of the EMP. In this model electrons and ions are initially created by MPI due to the light string, and a dipole moment is subsequently induced in the plasma via the radiation pressure force resulting from the velocity-dependent Lorentz force. For a laser field of frequency $\omega$ this force contains a second-harmonic component at $2 \omega$ in addition to a low frequency component, and it is this low frequency component of the force that creates the initial dipole moment in the plasma channel by longitudinally separating the light electrons from the heavy ions on the short time scale of the light string. Following the laser pulse the electron-ion system then starts to oscillate longitudinally at the plasma frequency 11 and generates an EMP propagating radially away from the plasma channel [10]. We stress that this mechanism for creating a dipole moment in a plasma is new and distinct from that due to the ponderomotive force from a tightly focused Gaussian beam [12,13]: In particular, the usual ponderomotive force acting on electrons is proportional to the spatial gradient of the applied light intensity [14]. For light strings of diameter $100 \mu \mathrm{m}$ and a few centimeters length the ponderomotive force should be negligible, and this was our motivation for looking for a new physical process that could create a dipole moment in the plasma channel.

In our model we consider a femtosecond laser pulse linearly polarized along the $\mathrm{x}$-axis, and propagating in air along the positive $z$-axis, with a central frequency $\omega$ in the IR region. For an electron plasma in the presence of an electric field $\vec{E}$ and a magnetic field $\vec{B}$, the hydrodynamic equation for the average velocity of an electron $\vec{v}$ reads 115]

$$
\frac{\partial \vec{v}}{\partial t}+(\overrightarrow{\mathrm{v}} \cdot \nabla) \overrightarrow{\mathrm{v}}=-\frac{e}{m}(\vec{E}+\eta \vec{v} \times \vec{B})+\left.\frac{\partial \vec{v}}{\partial t}\right|_{\text {scatt }},
$$

where $e$ and $m$ are the charge and mass of the electron, and $\left.(\partial \vec{v} / \partial t)\right|_{\text {scatt }}$ is the time rate of change of the velocity due to constituent particle scattering. The parameter $\eta$ is included to keep track of orders of perturbation theory and reflects the fact that the velocity-dependent Lorentz force is order $|\vec{v}| / c<<1$ smaller than the dominant force due to the electric field under the non-relativistic conditions appropriate here. To keep the theory as simple as possible we concentrate on the electron motion, assuming that the heavy ions remain stationary. In addition, we have the continuity equation for the electron density 4.15.

$$
\frac{\partial N_{e}}{\partial t}+\nabla \cdot\left(N_{e} \overrightarrow{\mathrm{v}}\right)=\sum_{K} \frac{\beta^{(K)}}{K \hbar \omega} I^{K}(\vec{r}, t),
$$

where $N_{e}$ is the electron density, $\omega$ is the central frequency of the input laser pulse, $K$ is the order of the MPI and the sum allows for the various constituents of air, mainly oxygen and nitrogen molecules, $\beta^{(K)}$ is the MPI coefficient derived from, for example, Keldysh theory 16], and $I(\vec{r}, t)$ is the intensity distribution of the laser pulse. The right-hand side of Eq. (2) describes the generation of electrons via MPI, since the effects of avalanche ionization for femtosecond pulses are negligible compared to MPI. To complete the system we couple these equations to the Maxwell equations for the electromagnetic field incorporating the effects of nonlinear 
self-focusing, plasma defocusing, and group velocity dispersion. The key Maxwell equation we need here is

$$
\nabla \cdot \vec{E}=-e\left(N_{e}-N_{i}\right) / \epsilon_{0},
$$

which determines the irrotational part of the electric field from the net charge density $-e\left(N_{e}-N_{i}\right) / \epsilon_{0}$, with $N_{i}$ the ion density of the plasma.

Equations (11-3) provide the basic dynamics of the plasma. Detailed numerical simulations of the formation and structure of light strings have been performed in a variety of situations [1 5 . Typically, light strings have a diameter of around $100 \mu \mathrm{m}$ and a temporal duration $t_{p} \approx 10 \mathrm{fs}$. Due to temporal compression associated with self-focusing of the input pulse [17], the duration $t_{p}$ of the light string is much shorter than the input pulse duration. Here we shall use electric and magnetic fields which reflect the characteristic properties of a single light string rather than explicitly solving for their detailed structure, which would obscure the physics to be presented.

To proceed we first consider the evolution of the electron velocity for short times on the order of the light string duration $t_{p}$ during which time the electrons are generated. For such short times we may safely neglect the effects of particle diffusion, hence we ignore spatial derivatives in Eq. (11). When the electrons are liberated via MPI they are accelerated in the laser field and undergo collisions, and we approximate the scattering as $\left.(\partial \vec{v} / \partial t)\right|_{\text {scatt }} \approx-\gamma \vec{v}$, with $\gamma \approx 10^{12}-10^{13} \mathrm{~s}^{-1}$ the collision rate for air at atmospheric pressure [10,18]. Furthermore, the dominant electromagnetic fields will be those due to the light string $\vec{E}=\vec{E}_{L}$ and $\vec{B}=\vec{B}_{L}$. The spatial dimensions of the light string and plasma channel are around $100 \mu \mathrm{m}^{2} \times 1 \mathrm{~cm}$, enormous on the scale of a cubic wavelength, and we approximate the fields as spatially uniform over the transverse profile of the plasma channel. The fields $\vec{E}_{L}$ and $\vec{B}_{L}$ due to the light string can then be expressed as

$$
\vec{E}_{L}(\vec{r}, t)=\frac{\hat{x}}{2}\left(\mathcal{E}_{L}\left(t-z / v_{g}\right) e^{i(k z-\omega t)}+c . c .\right),
$$

with $\vec{B}_{L}(\vec{r}, t)=\hat{y} E_{L} / c$, where $\mathcal{E}_{L}(\tau)=\sqrt{2 I_{0} / c \epsilon_{0}} \cdot e^{-\tau^{2} / t_{p}^{2}}$ is the Gaussian approximation to the light string electric field envelope with $\tau=t-z / v_{g}$ the retarded time in a reference frame moving at the light string group velocity $v_{g}, I_{0}$ and $t_{p}$ being the peak intensity and duration of the light string, $n_{0} \approx 1$ is the refractive-index of air, and $k=\omega / c$ is the magnitude of the optical wave vector. Since the light string is polarized along the $\mathrm{x}$-direction we expect the electron motion to be predominantly along the $\mathrm{x}$-axis, and we correspondingly write the electron velocity as $\vec{v}=\hat{x} v_{x}+\eta \hat{z} v_{z}$ : By symmetry the electron motion will be confined to the $\mathrm{x}-\mathrm{z}$ plane. Then to zeroth and first orders in the perturbation parameter $\eta$ Eq. (11) yields the pair of equations

$$
\begin{aligned}
\frac{\partial v_{x}}{\partial t} & =-\frac{e}{m} E_{L}-\gamma v_{x}, \\
\frac{\partial v_{z}}{\partial t} & =-\frac{e}{m}\left(\frac{v_{x}}{c}\right) E_{L}-\gamma v_{z} .
\end{aligned}
$$

Under the assumption that the electric field envelope $\mathcal{E}_{L}(\tau)$ varies slowly on the carrier time scale $\omega^{-1}$ the equation for $v_{x}$ may be solved approximately as

$$
v_{x}(z, t) \approx-\frac{e}{2 m}\left(\frac{\mathcal{E}_{L}\left(t-z / v_{g}\right) e^{i(k z-\omega t)}}{(\gamma-i \omega)}+c . c\right) .
$$

Next, inspection of the driving force term proportional to $-e\left(v_{x} / c\right) E_{L}$ appearing in Eq. (5) for $v_{z}$ reveals that it has high frequency or second-harmonic terms varying as $\pm 2 \omega$ in addition to a low frequency term. The low frequency term produces a force that is always directed along the positive z-axis, and creates a charge separation between the fast responding electrons and slow ions along the direction of the light string, and hence an initial dipole moment. In contrast, the charge separation induced by the second-harmonic term keeps switching direction, hence producing zero dipole moment on average, and we therefore isolate the low frequency component. Then by substituting Eqs. (4) and (6) into (5) for $v_{z}$ we obtain

$$
\begin{gathered}
\frac{\partial^{2} \xi}{\partial t^{2}}+\gamma \frac{\partial \xi}{\partial t}=\frac{F_{R P}}{m} \\
F_{R P}(z, t)=\frac{e^{2}}{2 m c} \frac{\gamma}{\left(\gamma^{2}+\omega^{2}\right)}\left|\mathcal{E}_{L}\left(t-z / v_{g}\right)\right|^{2},
\end{gathered}
$$

where $v_{z}=\partial \xi / \partial t, \xi(z, t)$ being the longitudinal $(z)$ electron-ion separation, and $F_{R P}$ is the radiation pressure force exerted on the electrons (see below). Integrating Eq. (7) over time and using $\xi(z,-\infty)=0$ and $\partial \xi /\left.\partial t\right|_{ \pm \infty}=0$ we obtain

$$
\xi_{0}=\frac{e^{2}}{\epsilon_{0} m^{2} \omega^{2} c^{2}} \int_{-\infty}^{\infty} I(\tau) d \tau
$$

where $I=\frac{1}{2} \epsilon_{0} c\left|\mathcal{E}_{L}\right|^{2}$ is the light string intensity, we have used $\omega>>\gamma$ to simplify the expression, and we have extended the upper time limit to infinity with the understanding that we formally only need a large enough time window to cover the light string duration.

The quantity $\xi_{0}$ is the longitudinal electron-ion separation induced by the light string, with associated dipole moment directed along the z-axis $d_{0}=-e \xi_{0}$, and is proportional to the time-integrated intensity or fluence of the light string, but relatively insensitive to the scattering rate so long as $\omega>>\gamma>0$. In general, $\xi_{0}$ could depend on position along the light string due to longitudinal variation of the intensity profile, but in keeping with our approximations we shall treat $\xi_{0}$ as a constant over the length of the plasma channel. Physically, the low frequency component of the Lorentz force exerts a force on each electron in the direction of propagation of 
the light string. To elucidate the physical origin of this force we realize that expression (7) for the radiation pressure force can be written as $F_{R P}=\hbar k \cdot(I / \hbar \omega) \cdot \sigma$, where $\hbar k$ is the momentum transferred to the electron along the z-axis per absorbed photon, $(I / \hbar \omega)$ is the photon flux (number of photons per unit area per unit time), and $\sigma=\left(k e^{2} \gamma / \omega m \epsilon_{0}\right) /\left(\gamma^{2}+\omega^{2}\right)$ is the cross-section for inverse Bremsstrahlung [19]. The force that causes the electron-ion separation along the direction of propagation of the light string may therefore be interpreted as the radiation pressure force that accompanies inverse Bremsstrahlung in which the electrons absorb photons in the field of the ions.

Our physical picture of the EMP generation is now as follows: The light string of duration $t_{p} \approx 10 \mathrm{fs}$ generates an initial dipole moment along the $\mathrm{z}$-axis in the plasma channel which subsequently oscillates at the plasma frequency $\omega_{p l}=\sqrt{N_{e} e^{2} / m \epsilon_{0}}$ 11,20 , and radiates an EMP radially outwards from the channel (see below). For electron densities $N_{e} \approx 10^{15} \mathrm{~cm}^{-3}$ typical of light string induced plasmas, we have $\omega_{p l}=2 \times 10^{12} \mathrm{rads}^{-1}$ with corresponding EMP wavelength $\lambda_{E M P}=2 \pi c / \omega_{p l} \approx 1$ $\mathrm{mm}$. Some other physical considerations are in order regarding this picture. In particular, in addition to the longitudinal electron-ion displacement $\xi_{0}$ induced by the light string the electrons also attain temperatures of the order of a few electron-volts [9] due to the oscillatory force $-e \vec{E}_{L}$ acting on them along the x-axis in the field of the light string. Physically, electron-electron collisions will cause the electron plasma, initially described by a non-equilibrium electron distribution, to relax towards an Maxwellian distribution which as a whole executes plasma oscillations along the z-axis [21]. We do not expect plasma oscillations along the $\mathrm{x}$-axis as the diameter of the plasma channel $(\approx 100 \mu \mathrm{m})$ is smaller than the EMP wavelength. The ionized electron-ion pairs can also undergo radiative recombination hence producing high frequency EM fields in addition to the relatively low frequency EMP of interest here.

To calculate the plasma-electron oscillations of the channel for times after the light string has passed we use the classic approach of Tonks and Langmuir [11]: Then Eq. (3) for the self-consistent electric field resulting from the charge separation becomes $\partial E_{z} / \partial z=$ $\left(e N_{e} / \epsilon_{0}\right)(\partial \xi / \partial z)$, with solution $E_{z}=\left(e N_{e} / \epsilon_{0}\right) \xi$. The longitudinal force on each electron is then $-e E_{z}=$ $m \partial \xi^{2} / \partial t^{2}$, which yields the following equation of motion for the electron-ion displacement

$$
\frac{\partial^{2} \xi}{\partial t^{2}}+\omega_{p l}^{2}(t) \xi=0
$$

where $\omega_{p l}(t)=\sqrt{N_{e}(t) e^{2} / m \epsilon_{0}}$ as before. Since the plasma density varies on a length scale $L$ of centimeters which is much larger than the anticipated EMP wavelength $\lambda_{E M P} \approx 1 \mathrm{~mm}$, we hereafter treat the plasma density, and hence the plasma frequency as constant over the plasma channel. Equation (9) is to be solved with the initial condition $\xi(0)=\xi_{0}$ from Eq. (8): This assumes that the initial electron-ion separation is not significantly degraded by thermalizing collisions, meaning that we are taking an upper bound for $\xi(0)$. For long times well after the light string has passed, the electron density decays due to radiative recombination as $\partial N_{e} / \partial t=-a N_{e}^{2}$ [8], which has the analytic solution $N_{e}(t)=N_{e}(0) /\left(1+a t N_{e}(0)\right)$. The initial value of the electron density can be calculated by integrating Eq. (2), but here we use typical value of $N_{e}(0)=10^{15} \mathrm{~cm}^{-3}$ obtained from the full numerical solutions 值5.

To obtain an approximate solution for the EMP due to the oscillating electron plasma we write the longitudinal current as $\vec{J}(\vec{r}, t)=-e N_{e}(\partial \xi / \partial t) \hat{z}$. Furthermore, since the plasma channel radius $r_{0} \approx 50 \mu \mathrm{m}$ is smaller than the EMP wavelength $\lambda_{E M P} \approx 1 \mathrm{~mm}$, and the plasma channel length $L>>\lambda_{E M P}$, we approximate the induced current in the channel as a line source

$$
\vec{J}(\vec{r}, t) \approx-e N_{e}(t) \dot{\xi}(t) \delta(x) \delta(y)\left(\pi r_{0}^{2}\right) \hat{z},
$$

where the dot signifies a time derivative. This current oscillating along the $\mathrm{z}$-axis will radiate a radially symmetric EMP $\vec{E}_{r a d}(\vec{r}, t)=\hat{z} E_{\text {rad }}(r, t)$, independent of $\mathrm{z}$ and dependent only on the radial distance $r$, that propagates through air and radially away from the plasma channel, and obeys Maxwell wave equation

$$
\left(\nabla^{2}-\frac{1}{c^{2}} \frac{\partial^{2}}{\partial t^{2}}\right) \vec{E}_{\text {rad }}(\vec{r}, t)=\mu_{0} \frac{\partial \vec{J}}{\partial t} .
$$

It is important in this model that the EMP radiates into the air surrounding the plasma channel as radiation at the plasma frequency $\omega_{p l}$ has zero group velocity and cannot therefore propagate in the plasma channel. Equation (11) along with the current in Eq. (10) can be solved using the Green's function for the wave equation 22

$E_{\text {rad }}(r, t)=\frac{e \mu_{0} N_{e}(t) r_{0}^{2}}{4} \int_{-\infty}^{\infty} d z \frac{\ddot{\xi}\left(t-\sqrt{r^{2}+z^{2}} / c\right)}{\sqrt{r^{2}+z^{2}}}$,

where we have taken the density $N_{e}(t)$ outside the integral based on the fact that it decays on a time scale of nanoseconds, slow compared to the plasma oscillations. Equation (12) shows that even though we have not included damping of the plasma oscillation the radiated EMP is limited in duration by the time it takes the electron plasma to recombine, which is on the order of a few nanoseconds [6 [10]: In contrast, Landau damping 20] of the plasma oscillations for our parameters occurred on a much longer time scale.

To illustrate the features of the EMP we have solved Eqs. (9) and (12) numerically with the initial condition (8). Here we chose the light string parameters $I_{0}=2 \times 10^{13} \mathrm{~W} / \mathrm{cm}^{2}, t_{p}=10 \mathrm{fs}$, an initial electron density $N_{e}(0)=10^{15} \mathrm{~cm}^{-3}$, and $a=5 \times 10^{-7} \mathrm{~cm}^{3} / \mathrm{s}$ [4]. We 
find that the radiated EMP has a peak field strength of $\left|E_{\text {max }}\right| \approx 2 \mathrm{kV} / \mathrm{m}$ at an observation distance $r=1 \mathrm{~mm}$ from the channel. The spectrum of the EMP is shown in Fig. (1), and the peak of the spectrum is at $\omega_{\text {peak }} \approx 10^{12}$ $\operatorname{rad} \mathrm{s}^{-1}$, which lies in the far IR region, which is consistent with the plasma frequency for a density around $N_{e} \approx 10^{15} \mathrm{~cm}^{-3}$. The Hertzian width of the EMP spectrum is around $\Delta \nu \approx 3 \times 10^{9} \mathrm{~s}^{-1}$, which is consistent with the inverse lifetime due to radiative electron recombination $a N_{e}(0) \approx 10^{9} \mathrm{~s}^{-1}$. Thus, the peak EMP frequency serves as a measure of the plasma density, and the width of the spectrum is a measure of the decay time of the electron plasma. This suggests that the EMP from light string induced plasma could serve as a useful diagnostic.

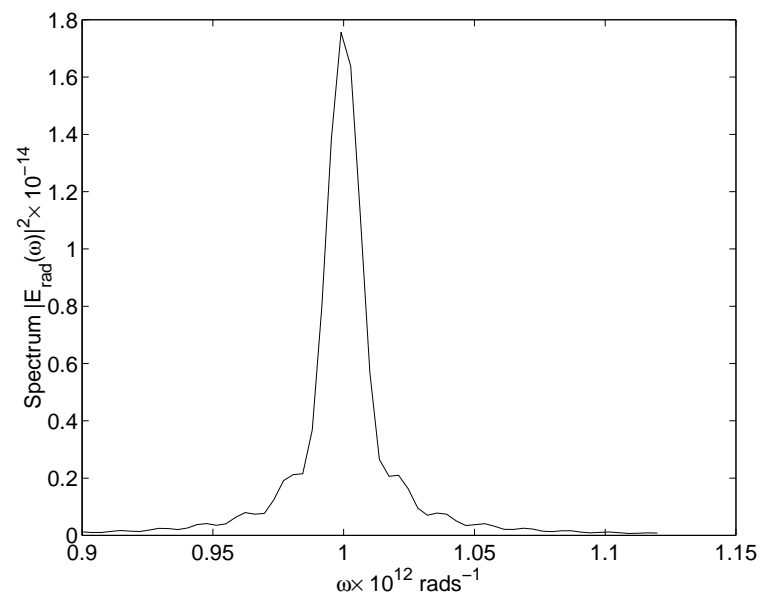

FIG. 1. Spectrum of the EMP.

In summary, we have shown that the EMP from a light string induced plasma channel can be understood as due to the establishment of a dipole moment in the channel by the radiation pressure force of the light string, which subsequently oscillates at the plasma frequency and radiates. Having established the basic physics, in future work we shall explore the details of the evolution of the plasma channel using the full kinetic theory 23,24, and in particular how the initial dipole moment dephases before radiating. This raises the prospect that the EMP field strength may be enhanced by creating $M$ parallel plasma channels [5] within the EMP wavelength, then the EMP intensity should be enhanced by $M^{2}$.

We are grateful to Profs. R. Binder and N. Bloembergen at the Optical Sciences Center, Prof. M. Bonitz of the University of Rostock, and Prof. S. Koch of the University of Marburg for useful discussions regarding this work. This work is sponsored by the U.S. Air Force Office of Scientific Research (AFOSR), under grants AFOSRF49620-00-1-002 and AFOSR-F49620-00-1-0190.
[1] A. Braun, G. Korn, X. Liu, D. Du, J. Squier, and G. Mourou, Opt. Lett. 20, 73 (1995).

[2] E. T. J. Nibbering, P. F. Curley, G. Grillon, B. S. Prade, M. A. Franco, F. Salin, and A. Mysyrowicz, Opt. Lett. 21, 62 (1996).

[3] A. Brodeur, C. Y. Chien, F. A. Ilkov, S. L. Chin, O. G. Kosareva, and V. P. Kandidov, Opt. Lett. 22, 304 (1997).

[4] M. Mlejnek, E. W. Wright,and J. V. Moloney, Opt. Lett. 23, 382 (1998).

[5] M. Mlejnek, M. Kolesik, J. V. Moloney, and E. W. Wright, Phys. Rev. Lett. 83, 2938 (1999).

[6] H. Schillinger, and R. Sauerbrey, Appl. Phys. B 68, 753 (1999).

[7] S. Tzortzakis, M. A. Franco, Y. -B. Andre, A. Chiron, B. Lamouroux, B. Prade, and A. Mysyrowicz, Phys. Rev. E 60, R3505 (1999).

[8] S. Tzortzakis, B. Prade, M. Franco, and A. Mysyrowicz, Opt. Comm. 181, 123 (2000).

[9] F. Vidal et. al. IEEE Trans. Plasma Sci. 28, 418 (2000).

[10] A. Proulx, A. Talebpour, S. Petit, S. L. Chin, Opt. Comm. 174, 305 (2000).

[11] L. Tonks and I Langmuir, Phys. Rev. 33, 195 (1929).

[12] H. Hamster, A. Sullivan, S. Gordon, W. White, and R. W. Falcone, Phys. Rev. Lett. 71, 2725 (1993).

[13] S. P. Goreslavsky and N. B. Narozhny, Nonl. Opt. Phys. Mat. 4, 799(1995).

[14] L. Landau and I. M. Lifshitz, Electrodynamics of Continuous Media (Pergamon, New York, 1960), Second Edition, pp. 59-64.

[15] N. Bloembergen, R. K. Chang, S. S. Jha, and C. H. Lee, Phys. Rev. 174, 813 (1968).

[16] L. V. Keldysh, Sov. Phys. JETP 20, 1307 (1965).

[17] G. L. McAllister, J. H. Marburger, and L. G. DeShazer, Phys. Rev. Lett. 21, 1648 (1968).

[18] J. F. Ready, Effects of high power laser radiation (Academic Press, New York, 1971) pp. 259-261.

[19] M. D. Feit and J. A. Fleck, Appl. Phys. Lett. 24, 170 (1975). These authors quote $\sigma=\left(k / n_{c}\right) \omega \tau /\left(1+\omega^{2} \tau^{2}\right)$, where in our notation $\tau \rightarrow 1 / \gamma$, and $n_{c} \rightarrow m \epsilon_{0} \omega^{2} / e^{2}$ is the electron density at which the plasma frequency equals $\omega$. With these changes one gets the formula for $\sigma$ quoted in the text.

[20] J. D. Jackson, Classical Electrodynamics (Wiley \& Sons, New York, 1975) Second Edition, Chap. 10.

[21] Private communications with Micha Bonitz (Univeristy of Rostock) and Stephan Koch (University of Marburg).

[22] See, for example, Mathews and Walker, Mathematical Methods of Physics (W. A. Benjamin, New York, 1965) Chap. 9, pp. 265-269.

[23] D. C. Scott, R. Binder, and S. W. Koch, Phys. Rev. Let. 69, 347 (1992).

[24] D. Kremp, Th. Bornath, M. Bonitz, and M. Schlanges, Phys. Rev. E 60, 4725 (1999). 